\title{
Rotational superradiant scattering in a vortex flow
}

Theo Torres ${ }^{1}$, Sam Patrick ${ }^{1}$, Antonin Coutant ${ }^{1}$, Maurício Richartz ${ }^{2}$, Edmund W. Tedford ${ }^{3} \&$ Silke Weinfurtner ${ }^{1,4,5}$

${ }^{1}$ School of Mathematical Sciences, University of Nottingham, University Park, Nottingham, NG7 $2 R D, U K$

${ }^{2}$ CMCC - Centro de Matemática, Computação e Cognição, Universidade Federal do ABC (UFABC), 09210-170 Santo André, São Paulo, Brazil

${ }^{3}$ Department of Civil Engineering, University of British Columbia, 6250 Applied Science Lane, Vancouver, Canada V6T $1 Z 4$

${ }^{4}$ School of Physics and Astronomy, University of Nottingham, Nottingham, NG7 2RD, UK.

${ }^{5}$ Centre for the Mathematics and Theoretical Physics of Quantum Non-Equilibrium Systems, University of Nottingham, Nottingham, NG7 2RD, UK.

When an incident wave scatters off of an obstacle, it is partially reflected and partially trans- $\quad 1$ mitted. In theory, if the obstacle is rotating, waves can be amplified in the process, extracting 2 energy from the scatterer. Here we describe in detail the first laboratory detection of this 3 phenomenon, known as superradiance ${ }^{1-4}$. We observed that waves propagating on the sur- 4 face of water can be amplified after being scattered by a draining vortex. The maximum 5 amplification measured was $14 \% \pm 8 \%$, obtained for $3.70 \mathrm{~Hz}$ waves, in a $6.25 \mathrm{~cm}$ deep fluid, 6 in consistency with superradiant scattering caused by rapid rotation. Our experimental find- 7 ings will shed new light not only on Black Hole Physics, since shallow water waves scattering 8 
on a draining fluid constitute an analogue of a black hole ${ }^{\mathbf{5 - 1 0}}$, but also on hydrodynamics, 9 due to its close relation to over-reflection instabilities ${ }^{11-13}$.

In water, perturbations of the free surface manifest themselves by a small change $\xi(t, \mathbf{x}) \quad 11$ of the water height. On a flat bottom, and in the absence of flow, linear perturbations are well 12 described by superpositions of plane waves of definite frequency $f(\mathrm{~Hz})$ and wave-vector $\mathbf{k}(\mathrm{rad} / \mathrm{m}) . \quad 13$ When surface waves propagate on a changing flow, the surface elevation is generally described by 14 the sum of two contributions $\xi=\xi_{I}+\xi_{S}$, where $\xi_{I}$ is the incident wave produced by a source, e.g. a 15 wave generator, while $\xi_{S}$ is the scattered wave, generated by the interaction between the incident 16 wave and the background flow. In this work, we are interested on the properties of this scattering 17 on a draining vortex flow which is assumed to be axisymmetric and stationary. At the free surface, 18 the velocity field is given in cylindrical coordinates by $\mathbf{v}=v_{r} \mathbf{e}_{r}+v_{\theta} \mathbf{e}_{\theta}+v_{z} \mathbf{e}_{z}$.

Due to the symmetry, it is appropriate to describe $\xi_{I}$ and $\xi_{S}$ using polar coordinates $(r, \theta)$. 20 Any wave $\xi(t, r, \theta)$ can be decomposed into partial waves ${ }^{10,14}$,

$$
\xi(t, r, \theta)=\operatorname{Re}\left[\sum_{m \in \mathbb{Z}} \int_{0}^{\infty} \varphi_{f, m}(r) \frac{e^{-2 i \pi f t+i m \theta}}{\sqrt{r}} d f\right]
$$

where $m \in \mathbb{Z}$ is the azimuthal wave number and $\varphi_{f, m}(r)$ denotes the radial part of the wave. Each 22 component of this decomposition has a fixed angular momentum proportional to $m$, instead of a 23 fixed wave-vector k. (To simplify notation, we drop the indices $f_{f, m}$ in the following.) Since the ${ }_{24}$ background is stationary and axisymmetric, waves with different $f$ and $m$ propagate independently. $\quad 25$ Far from the centre of the vortex, the flow is very slow, and the radial part $\varphi(r)$ becomes a sum of ${ }_{26}$ 
oscillatory solutions,

$$
\varphi(r)=A_{\text {in }} e^{-i k r}+A_{\text {out }} e^{i k r},
$$

where $k=\|\mathbf{k}\|^{2}$ is the wave-vector norm. This describes the superposition of an inward wave of ${ }_{28}$ (complex) amplitude $A_{\text {in }}$ propagating towards the vortex, and an outward one propagating away ${ }_{29}$ from it with amplitude $A_{\text {out }}$. These coefficients are not independent. The $A_{\text {in }}$ 's, one for each $f \quad{ }_{30}$ and $m$ component, are fixed by the incident part $\xi_{I}$. If the incident wave is a plane wave $\xi={ }_{31}$ $\xi_{0} e^{-2 i \pi f t+i \mathbf{k} \cdot \mathbf{x}}$, then the partial amplitudes are given by $A_{\text {in }}=\xi_{0} e^{i m \pi+i \pi / 4} / \sqrt{2 \pi k}$. In other words, $\quad 32$ a plane wave is a superposition containing all azimuthal waves, something that we have exploited ${ }_{33}$ in our experiment. On the contrary, $A_{\text {out }}$ depends on the scattered part $\xi_{S}$, and how precisely the ${ }_{34}$ waves propagate in the centre and interact with the background vortex flow. In the limit of small 35 amplitudes, there is a linear relation between the $A_{\text {in }}$ 's and $A_{\text {out }}$ 's, and by the symmetries of the ${ }_{36}$ flow, different $f$ and $m$ decouple ${ }^{10,15}$.

This allows us to define the reflection coefficient at fixed $f$ and $m$ as the ratio between the ${ }_{38}$ outward $\left(J_{\text {out }}\right)$ and inward $\left(J_{\text {in }}\right)$ energy fluxes,

$$
R=\sqrt{\frac{J_{\text {out }}}{J_{\text {in }}}} .
$$

In the linear approximation, the wave energy is a quadratic quantity in wave amplitude, and $R$ is 40 proportional to the amplitude ratio $\left|A_{\text {out }} / A_{\text {in }}\right|$.

If $|R|<1$, the wave has lost energy during the scattering, and hence has undergone absorp- 42 tion. In this work we show experimentally that, under certain conditions, the reflection coefficient 43 satisfies $|R|>1$. We further argue that the amplified wave has extracted rotational energy from ${ }_{44}$ 
the vortex during the process.

We conducted our experiment in a $3 \mathrm{~m}$ long and $1.5 \mathrm{~m}$ wide rectangular water tank. Water ${ }_{46}$ is pumped continuously in from one end corner, and is drained through a hole $\left(4 \mathrm{~cm}\right.$ in diameter) ${ }_{47}$ in the middle. The water flows in a closed circuit. We first establish a stationary rotating draining 48 flow by setting the flow rate of the pump to $37.5 \pm 0.5 \ell / \mathrm{min}$ and waiting until the depth (away 49 from the vortex) is steady at $6.25 \pm 0.05 \mathrm{~cm}$. We then generate plane waves from one side of 50 the tank, with an excitation frequency varying from $2.87 \mathrm{~Hz}$ to $4.11 \mathrm{~Hz}$. On the side of the tank 51 opposite the wave generator, we have placed an absorption beach (we have verified that the amount 52 of reflection from the beach is below $5 \%$ in all experiments). We record the free surface with a 53 high speed 3D air-fluid interface sensor. The sensor is a joint-invention ${ }^{16}$ (patent No. DE $102015 \quad 54$ 001365 A1) between The University of Nottingham and EnShape GmbH (Jena, Germany). $\quad 55$

Using this data, we apply two filters. We first perform a Fourier transform in time, in order 56 to single out the signal at the excitation frequency $f_{0}$. This allows us to filter out the (stationary) 57 background height, lying at $f=0$, as well as the high frequency noise. Moreover, we observe 58 that the second harmonic, at $2 f_{0}$, is also excited by the wave generator. This gives us an upper 59 bound on the amount of nonlinearity of the system. In all experiments, the relative amplitude 60 of the second harmonic compared to the fundamental stays below $14 \%$. The obtained pattern 61 shows a stationary wave of frequency $f_{0}$ scattering on the vortex, which consists of the interfering 62 superposition of the incident wave $\xi_{I}$ with the scattered one $\xi_{S}$. This pattern is shown on Fig. 1 for $\quad 63$ various frequencies, and looks very close to what was predicted on theoretical grounds for simple $\quad 64$ 
bathtub flow models ${ }^{10,17}$. We also observe that incident waves have more wave fronts on the upper 65 half of the vortex in comparison with the lower half - see the various wave characteristics in panels 66 (A-F) in Fig. 1. This angular phase shift is analogous to the Aharonov-Bohm effect, and has been 67 observed in previous water wave experiments ${ }^{18,19}$. Our detection method allows for a very clear 68 visualization of this effect.

The second filter is the polar Fourier transform, which selects a specific azimuthal wave num- $\quad 70$ ber $m$, and allows the radial profile $\varphi(r)$ to be determined. To extract the reflection coefficient, we 71 use a windowed Fourier transform of the radial profile $\varphi(r)$. The windowing is done on the inter- 72 val $\left[r_{\min }, r_{\max }\right]$. When $r_{\min }$ is large enough, the radial profile $\varphi$ contains two Fourier components 73 [see Eq. (2)], one of negative $k$ (inward wave), and one of positive $k$ (outward wave). The ratio 74 between their two amplitudes gives us the reflection coefficient (up to the energy correction, see 75 Methods - Wave energy). In order to better resolve the two peaks, we have applied a Hamming 76 window on the radial profile over the interval $\left[r_{\min }, r_{\max }\right]$. In all experiments, $r_{\min } \simeq 0.15 \mathrm{~m}$, while $\quad 77$ $r_{\max } \simeq 0.39 \mathrm{~m}$. We also point out that the minimum radius such that the radial profile reduces to 78 Eq. (2) increases with $m$. With the size of our window, and the wavelength range of the experiment, 79 we can resolve with confidence $m=-2,-1,0,1,2$.

On Fig. 2 we represent, for several azimuthal numbers $m$, the absolute value of the reflection $\quad 81$ coefficient $R$ as a function of the frequency $f$. We observe two distinct behaviours, depending 82 on the sign of $m$. Negative $m$ 's (waves counterrotating with the vortex) have a low reflection ${ }_{83}$ coefficient, which means that they are essentially absorbed in the vortex hole. On the other hand, 84 
positive $m$ 's have a reflection coefficient close to 1 . In some cases this reflection is above one, 85 meaning that the corresponding mode has been amplified while scattering on the vortex. To confirm 86 this amplification we have repeated the same experiment 15 times at the frequency $f=3.8 \mathrm{~Hz}$ and $\quad 87$ water height $h_{0}=6.25 \pm 0.05 \mathrm{~cm}$, for which the amplification was the highest. We present the 88 result on Fig. 3. On this figure we clearly observe that the modes $m=1$ and $m=2$ are amplified 89 by factors $R_{m=1} \sim 1.09 \pm 0.03$, and $R_{m=2} \sim 1.14 \pm 0.08$ respectively. On Figs. 2 and 3, we have 90 also shown the reflection coefficients obtained for a plane wave propagating on standing water 91 of the same depth. Unlike what happens in the presence of a vortex, the reflection coefficients 92 are all below 1 (within error bars). For low frequencies it is close to 1 , meaning that the wave is ${ }_{93}$ propagating without losses, while for higher frequencies it decreases due to a loss of energy during 94 the propagation, i.e. damping.

The origin of this amplification can be explained by the presence of negative energy waves 96 20,21. Negative energy waves are excitations that lower the energy of the whole system (i.e. back- $\quad 97$ ground flow and excitation) instead of increasing it. In our case, the sign of the energy of a wave is 98 given by the angular frequency in the fluid frame $\omega_{\text {fluid }}$. If the fluid rotates with an angular velocity 99 $\Omega(r)$, in $\mathrm{rad} / \mathrm{s}$, we have $\omega_{\text {fluid }}=2 \pi f-m \Omega(r)$. At fixed frequency, when the fluid rotates fast 100 enough, the energy becomes negative. If part of the wave is absorbed in the hole, carrying negative 101 energy, the reflected part must come out with a higher positive energy to ensure conservation of the 102 total energy ${ }^{2}$. Using Particle Imaging Velocimetry (PIV), we have measured the velocity field of 103 the vortex flow of our experiment. As we see on Fig. 4A, close to the centre, the angular velocity 104 is quite high, and the superradiant condition $2 \pi f<m \Omega$ is therefore satisfied for our frequency 105 
range.

Our experiment demonstrates that a wave scattering on a rotating vortex flow can carry away $\quad 107$ more energy than the incident wave brings in. Our results show that the phenomenon of superradi- 108 ance is very robust and requires few ingredients to occur, namely high angular velocities, allowing 109 for negative energy waves, and a mechanism to absorb these negative energies. For about half of 110 the frequency range, our results confirm superradiant amplification despite a significant damping 111 of the waves. The present experiment does not reveal the mechanism behind the absorption of the 112 negative energies. The likely possibilities are that they are dissipated away in the vortex throat, 113 in analogy to superradiant cylinders ${ }^{4,22}$, that they are trapped in the hole ${ }^{23}$ and unable to escape, 114 similarly to what happens in black holes ${ }^{24,25}$, or a combination of both. A possible way to dis- 115 tinguish between the two in future experiments would be to measure the amount of energy going 116 down the throat. 

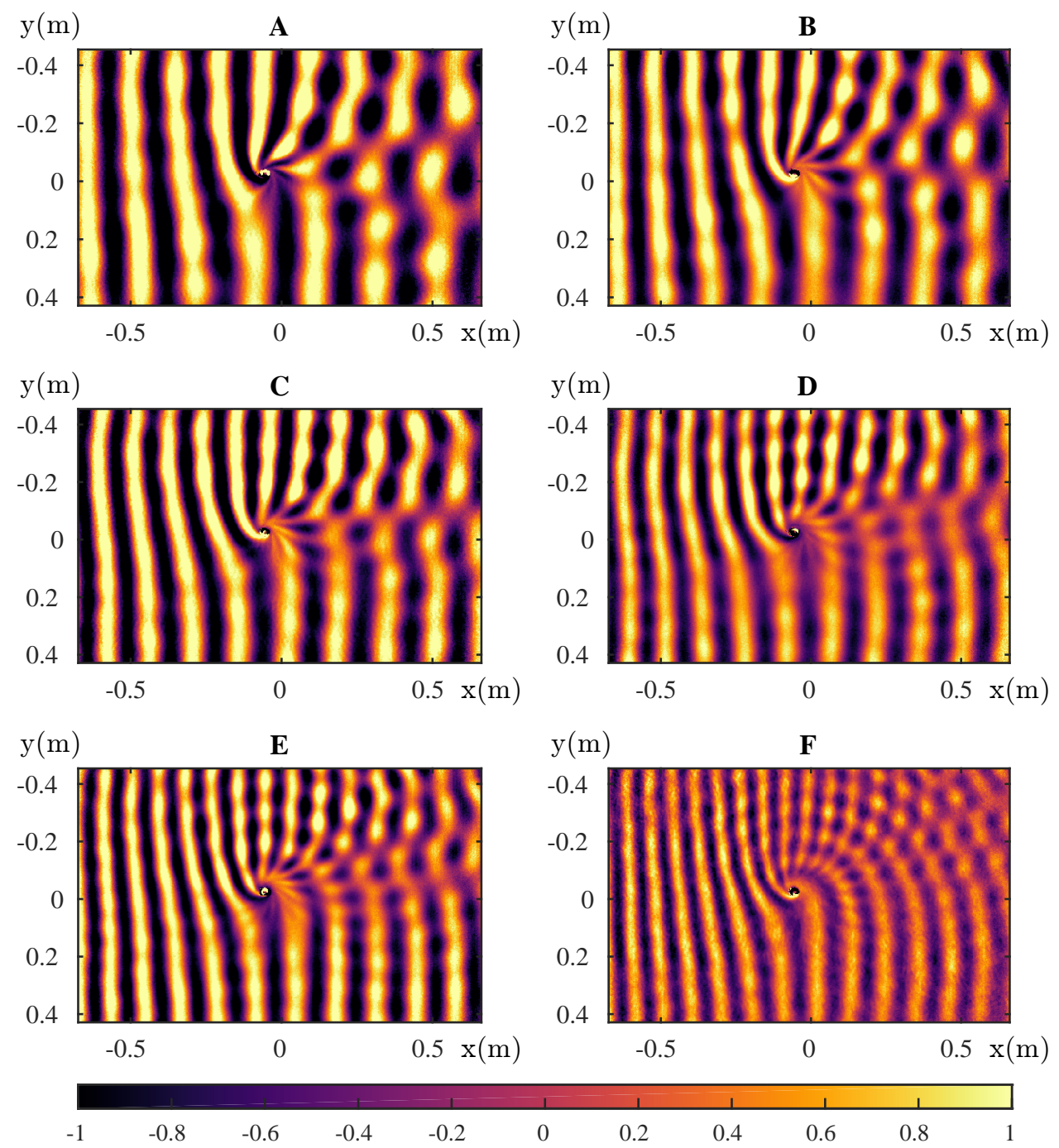

Figure 1 | Wave characteristics of the surface perturbation $\xi$, filtered at a single frequency, for six 119 different frequencies. The frequencies are $2.87 \mathrm{~Hz}(\mathbf{A}), 3.04 \mathrm{~Hz}(\mathbf{B}), 3.27 \mathrm{~Hz}$ (C), $3.45 \mathrm{~Hz}$ (D), 120 $3.70 \mathrm{~Hz}(\mathbf{E})$, and $4.11 \mathrm{~Hz}(\mathbf{F})$. The horizontal and vertical axis are in metres (m), while the color ${ }_{121}$ scale is in millimetres $(\mathrm{mm})$. The patterns show the interfering sum of the incident wave with the ${ }_{122}$ scattered one. The waves are generated on the left side and propagate to the right across the vortex $\quad 123$ centred at the origin. 


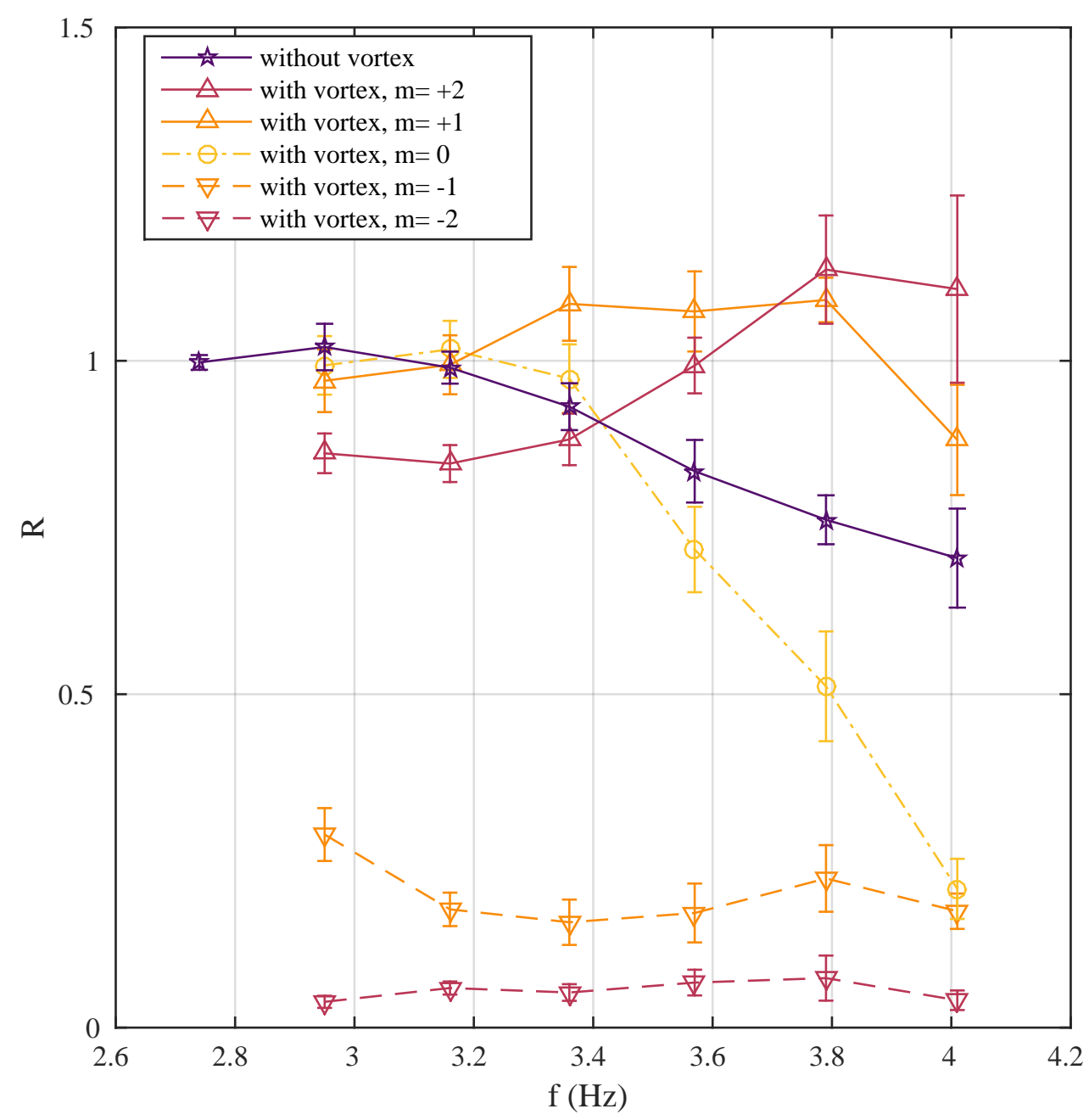

Figure $2 \mid$ Reflection coefficients for various frequencies and various $m$ 's. For the vortex experi- ${ }_{126}$ ments the statistical average is taken over 6 repetitions, except for $f=3.70 \mathrm{~Hz}$ where we have $15 \quad 127$ repetitions. The purple line (star points) shows the reflection coefficients of a plane wave in stand- ${ }_{128}$ ing water of the same height. We observe a significant damping for the frequencies above $3 \mathrm{~Hz}$ (see ${ }_{129}$ Fig. 2). In future experiments, we hope to reduce this damping by working with purer water ${ }^{26} \cdot{ }_{130}$ Each point of a plane wave on standing water (i.e. without vortex) is averaged over 5 repetitions ${ }_{131}$ 
instead of 6 , and over $m=-2 \ldots 2$ (the reflection coefficient of a plane wave on standing water is 132 in theory independent of $m$, see also Fig. 3). The errors bars indicate the standard deviation over ${ }_{133}$ these experiments, the energy uncertainty and the standard deviation over several centre choices $\quad 134$ (see Methods). The main contribution comes from the variability of the value of the reflection ${ }_{135}$ coefficient for different repetitions of the experiment. We have also extracted the signal-to-noise ${ }_{136}$ ratio for each experiment, and its contribution to the error bars is negligible (see Method - Data ${ }_{137}$ $\begin{array}{ll}\text { Analysis). } & 138\end{array}$ 


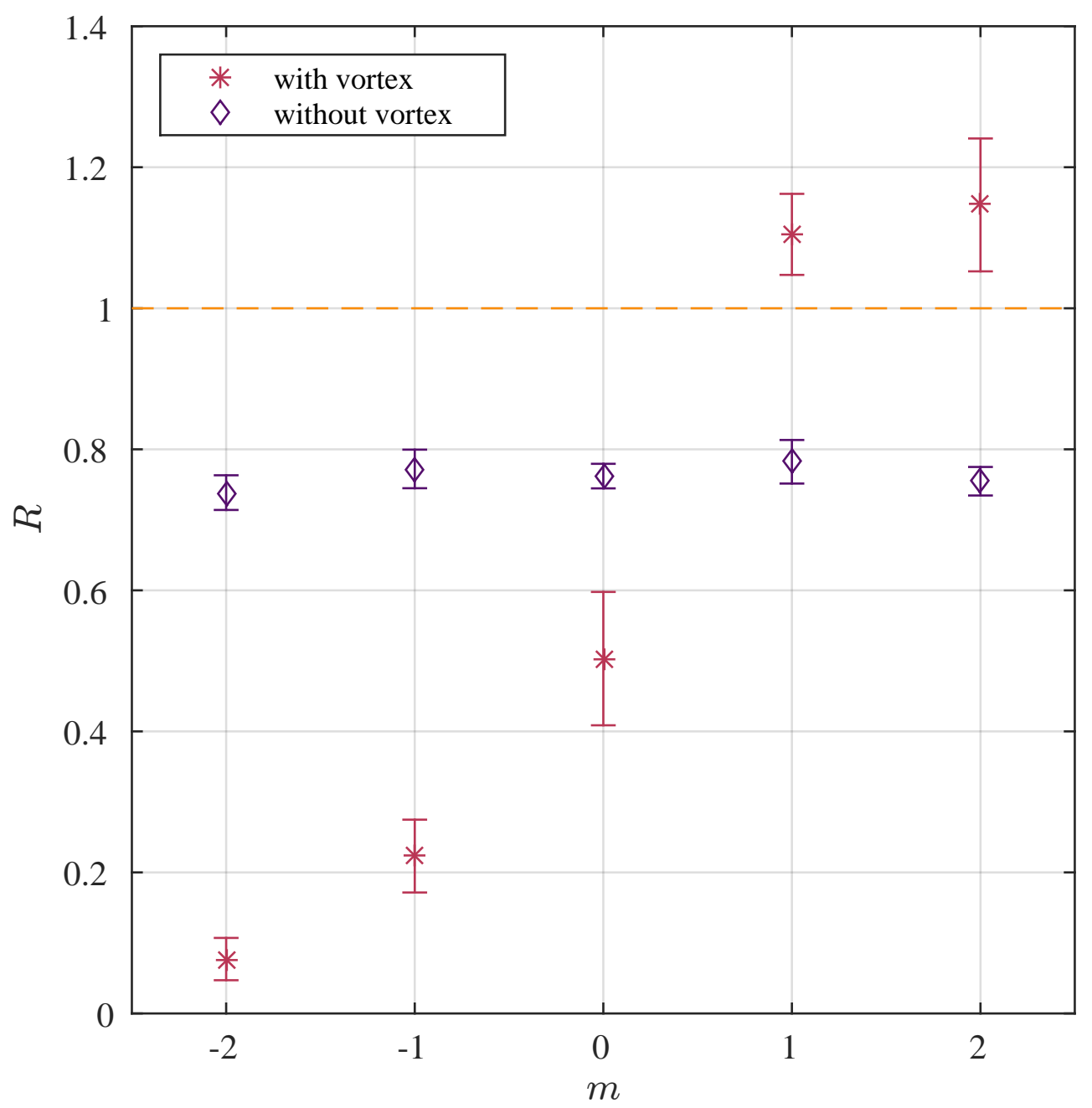

Figure $3 \mid$ Reflection coefficients for different $m$ 's, for the frequency $f=3.70 \mathrm{~Hz}$ (stars). We ${ }_{140}$ have also shown the reflection coefficients for plane waves without a flow, at the same frequency 141 and water height (diamonds). We see that the plane wave reflection coefficients are identical for all 142 $m$ 's, and all below 1 (within error bars). The statistic has been realized over 15 experiments. Error ${ }_{143}$ bars include the same contributions as in Fig. 2. 

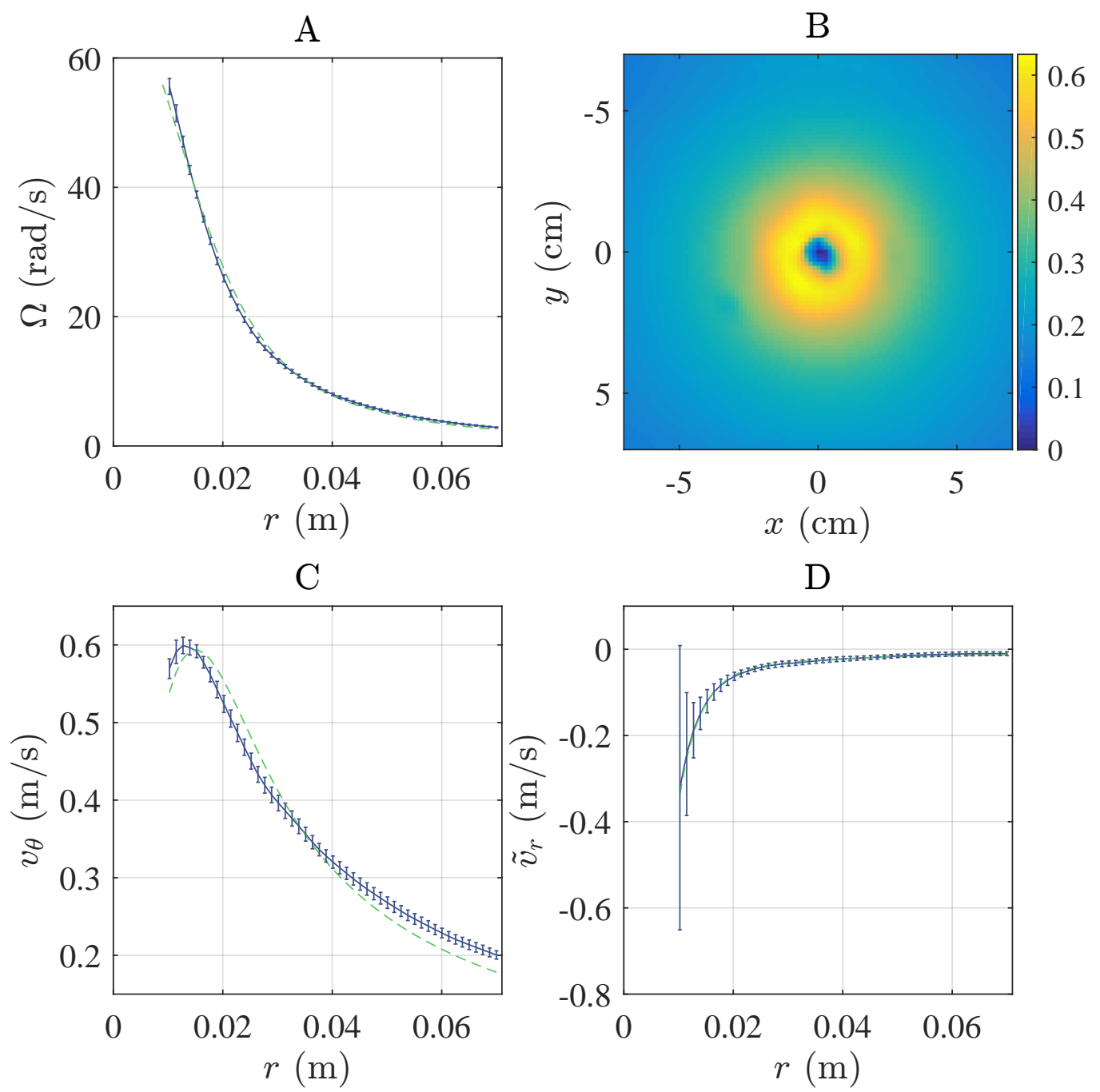

Figure 4 | PIV measurements of the velocity field averaged of 10 experiments. (A) Angular fre- ${ }_{146}$ quency profile as a function of $r$. (B) Norm of the velocity field of the background flow (in m/s). ${ }_{147}$ (C) $v_{\theta}$ profile as function of $r$. (D) $\tilde{v}_{r}$ profile as function of $r$ (see Methods - PIV measurements). 148 The profiles are fitted with a model of the Lamb vortex type in equation (9), dashed-green line. 149 The error bars correspond to standard deviations across the 10 measurements. 
1. Brito, R., Cardoso, V. \& Pani, P. Superradiance. Lect. Notes Phys. 906, 1-237 (2015).

2. Richartz, M., Weinfurtner, S., Penner, A. J. \& Unruh, W. G. Generalized superradiant scatter- ${ }^{153}$ ing. Phys. Rev. D 80, 124016 (2009).

3. Zel'Dovich, Y. B. Generation of Waves by a Rotating Body. JETP Lett. 14, 180-181 (1971). ${ }^{155}$

4. Zel'Dovich, Y. B. Amplification of Cylindrical Electromagnetic Waves Reflected from a Ro- ${ }_{156}$ tating Body. Sov. Phys. JETP 35, 1085-1087 (1972).

5. Unruh, W. Experimental black hole evaporation. Phys. Rev. Lett. 46, 1351-1353 (1981).

6. Visser, M. Acoustic black holes: horizons, ergospheres and Hawking radiation. Classical and 159 Quantum Gravity 15, 1767-1791 (1998).

7. Schützhold, R. \& Unruh, W. G. Gravity wave analogues of black holes. Phys. Rev. D 66, 161 044019 (2002).

8. Weinfurtner, S., Tedford, E. W., Penrice, M. C. J., Unruh, W. G. \& Lawrence, G. A. Measure- ${ }^{163}$ ment of stimulated hawking emission in an analogue system. Phys. Rev. Lett. 106, $021302 \quad 164$ (2011).

9. Steinhauer, J. Observation of thermal Hawking radiation and its entanglement in an analogue 166 black hole. Nature Phys. 12, 959-965 (2016).

10. Dolan, S. R. \& Oliveira, E. S. Scattering by a draining bathtub vortex. Phys. Rev. D87, 124038168 (2013). 
11. Acheson, D. J. On over-reflexion. Journal of Fluid Mechanics 77, 433-472 (1976).

12. Kelley, D. H., Triana, S. A., Zimmerman, D. S., Tilgner, A. \& Lathrop, D. P. Inertial waves 171 driven by differential rotation in a planetary geometry. Geophysical and Astrophysical Fluid 172 Dynamics 101, 469-487 (2007).

13. Fridman, A. et al. Over-reflection of waves and over-reflection instability of flows revealed in 174 experiments with rotating shallow water. Physics Letters A 372, 4822-4826 (2008).

14. Newton, R. G. Scattering theory of waves and particles (Courier Dover Publications, 1982). 176

15. Richartz, M., Prain, A., Liberati, S. \& Weinfurtner, S. Rotating black holes in a draining ${ }_{177}$ bathtub: superradiant scattering of gravity waves. Phys. Rev. D 91, 124018 (2015).

16. Schaffer, M., Große, M. \& Weinfurtner, S. Verfahren zur 3d-vermessung von flüssigkeiten und 179 gelen (2016). URL http://google.com/patents/DE102015001365A1?cl=zh. ${ }_{180}$ DE Patent App. DE201,510,001,365.

17. Dolan, S. R., Oliveira, E. S. \& Crispino, L. C. B. Aharonov-Bohm effect in a draining bathtub 182 vortex. Physics Letters B 701, 485-489 (2011).

18. Berry, M., Chambers, R., Large, M., Upstill, C. \& Walmsley, J. Wavefront dislocations in the 184 aharonov-bohm effect and its water wave analogue. European Journal of Physics 1, 154-162 185 (1980).

19. Vivanco, F., Melo, F., Coste, C. \& Lund, F. Surface wave scattering by a vertical vortex and the 187 symmetry of the aharonov-bohm wave function. Phys. Rev. Lett. 83, 1966-1969 1966 (1999). 188 
20. Stepanyants, Y. A. \& Fabrikant, A. Propagation of waves in shear flows. Physical and Math- 189 ematical Literature Publishing Company, Russian Academy of Sciences, Moscow (1996). 190

21. Coutant, A. \& Parentani, R. Undulations from amplified low frequency surface waves. Phys. 191 Fluids 26, 044106 (2014).

22. Cardoso, V., Coutant, A., Richartz, M. \& Weinfurtner, S. Detecting rotational superradiance 193 in fluid laboratories. Phys. Rev. Lett. 117, 271101 (2016).

23. Basak, S. \& Majumdar, P. 'Superresonance' from a rotating acoustic black hole. Class. Quant. ${ }^{195}$ Grav. 20, 3907-3914 (2003).

24. Misner, C. Stability of Kerr black holes against scalar perturbations. Bulletin of the American 197 Physical Society 17, 472 (1972).

25. Starobinsky, A. A. Amplification of waves during reflection from a rotating black hole. Sov. 199 Phys. JETP 37, 28-32 (1973).

26. Przadka, A., Cabane, B., Pagneux, V., Maurel, A. \& Petitjeans, P. Fourier transform profilom- 201 etry for water waves: how to achieve clean water attenuation with diffusive reflection at the 202 water surface? Experiments in fluids 52, 519-527 (2012). fields. Class.Quant.Grav. 30, 085009 (2013). 
29. Coutant, A. \& Weinfurtner, S. The imprint of the analogue Hawking effect in subcritical flows. 207

Phys. Rev. D 94, 064026 (2016).

Wave energy. To verify that the observed amplification increases the energy of the wave, we 210 compare the energy current of the inward wave with respect to the outward one. Since energy is 211 transported by the group velocity $v_{g}$, the energy current is given by $J=g \omega_{\text {fluid }}^{-1} v_{g}|A|^{2} / f$ (up to 212 the factor $1 / f$, this is the wave action, an adiabatic invariant of waves ${ }^{27-29}$ ). If the background ${ }^{213}$ flow velocity is zero, then the ratio $J_{\text {out }} / J_{\text {in }}$ is simply $\left|A_{\text {out }} / A_{\text {in }}\right|^{2}$. However, in the presence of the 214 vortex, we observe from our radial profiles $\varphi(r)$ [defined in equation (1)] that the wave number 215 of the inward and outward waves are not exactly opposite. The origin of this (small) difference is 216 that the flow velocity is not completely negligible in the observation window. It generates a small 217 Doppler shift that differs depending on whether the wave propagates against or with the flow. In 218 this case, the ratio of the energy currents picks up a small correction with respect to the ratio of the ${ }_{219}$ amplitudes, namely,

$$
\frac{J_{\text {out }}}{J_{\text {in }}}=\left|\frac{\omega_{\text {fluid }}^{\text {in }} v_{g}^{\text {out }}}{\omega_{\text {fluid }}^{\text {out }} v_{g}^{\text {in }}}\right|\left|\frac{A_{\text {out }}}{A_{\text {in }}}\right|^{2} .
$$

To estimate this factor, we assume that the flow varies slowly in the observation window, such 221 that $\omega_{\text {fluid }}$ obeys the usual dispersion relation of water waves, $\omega_{\text {fluid }}^{2}=g k \tanh \left(h_{0} k\right)$. (This 222 amounts to a WKB approximation, and capillarity is neglected.) Under this assumption, the ${ }_{223}$ group velocity is the sum of the group velocity in the fluid frame, given by the dispersion rela- 224 tion, $v_{g}^{\text {fluid }}=\partial_{k} \sqrt{g k \tanh \left(h_{0} k\right)}$, and the radial velocity of the flow $v_{r}$. Hence the group velocity ${ }_{225}$ 
needed for the energy ratio (4) splits into two: $v_{g}=v_{g}^{\text {fluid }}+v_{r}$. The first term is obtained only with 226 the values of $k_{\text {in }}$ and $k_{\text {out }}$, extracted from the radial Fourier profiles. The second term requires the ${ }_{227}$ value of $v_{r}$, which we do not have to a sufficient accuracy. However, using the PIV data, we see 228 that the contribution of this last term amounts to less than $1 \%$ in all experiments (this uncertainty ${ }_{229}$ is added to the error bars on Figs. 2 and 3).

Data analysis. We record the free surface of the water in a region of $1.33 \mathrm{~m} \times 0.98 \mathrm{~m}$ over the ${ }_{231}$ vortex during 13.2 s. From the sensor we obtain 248 reconstructions of the free surface. These ${ }_{232}$ reconstructions are triplets $X_{i j}, Y_{i j}$ and $Z_{i j}$ giving the coordinates of $640 \times 480$ points on the ${ }_{233}$ free surface. Because of the shape of the vortex, and noise, parts of the free surface cannot be ${ }_{234}$ seen by our sensor, resulting in black spots on the image. Isolated black spots are corrected by 235 interpolating the value of the height using their neighbours. This procedure is not possible in the ${ }_{236}$ core of the vortex and we set these values to zero.

To filter the signal in frequency, we first crop the signal in time so as to keep an integer 238 number of cycles to reduce spectral leakage. We then select a single frequency corresponding to 239 the excitation frequency $f_{0}$. After this filter, we are left with a 2-dimensional array of complex 240 values, encoding the fluctuations of the water height $\xi\left(X_{i j}, Y_{i j}\right)$ at the frequency $f_{0} . \xi\left(X_{i j}, Y_{i j}\right)$ is ${ }_{241}$ defined on the grids $X_{i j}$, and $Y_{i j}$, whose points are not perfectly equidistant (this is due to the fact 242 that the discretization is done by the sensor software in a coordinate system that is not perfectly 243 parallel to the free surface).

To select specific azimuthal numbers, we convert the signal from cartesian to polar coordi- ${ }_{245}$ 
nates. For this we need to find the centre of symmetry of the background flow. We define our 246 centre to be the centre of the shadow of the vortex, averaged over time (the fluctuations in time are 247 smaller than a pixel). To verify that this choice does not affect the end result, we performed a sta- ${ }_{248}$ tistical analysis on different centre choices around this value, and added the standard deviation to 249 the error bars. Once the centre is chosen, we perform a discrete Fourier transform on the irregular 250 grid $\left(X_{i j}, Y_{i j}\right)$. We create an irregular polar grid $\left(r_{i j}, \theta_{i j}\right)$ and we compute 251

$$
\varphi_{m}\left(r_{i j}\right)=\frac{\sqrt{r_{i j}}}{2 \pi} \sum_{j} \xi\left(r_{i j}, \theta_{i j}\right) e^{-i m \theta_{i j}} \Delta \theta_{i j}
$$

where $\Delta \theta_{i j}=\left(\Delta X_{i j} \Delta Y_{i j}\right) /\left(r_{i j} \Delta r_{i j}\right)$ is the line element along a circle of radius $r_{i j}$.

To extract the inward and outward amplitudes $A_{\text {in }}$ and $A_{\text {out }}$, we compute the radial Fourier ${ }_{253}$ transform $\tilde{\varphi}_{m}(k)=\int \varphi_{m}(r) e^{-i k r} d r$ over the window $\left[r_{\min }, r_{\max }\right]$. Due to the size of the window ${ }^{254}$ compared to the wavelength of the waves, we can only capture a few oscillations in the radial 255 direction, typically between 1 and 3 . This results in broad peaks around the values $k_{\text {in }}$ and $k_{\text {out }}{ }_{256}$ of the inward and outward components. We assume that these peaks contain only one wavelength 257 (no superposition of nearby wavelengths), which is corroborated by the fact that we have filtered 258 in time, and the dispersion relation imposes a single wavelength at a given frequency. To reduce ${ }^{259}$ spectral leakage, we use a Hamming window function on $\left[r_{\min }, r_{\max }\right]$, defined as 260

$$
W(n)=0.54-0.46 \cos \left(2 \pi \frac{n}{N}\right)
$$

where $n$ is the pixel index running from 1 to $N$. This window is optimized to reduce the secondary ${ }_{261}^{261}$ lobe, and allows us to better distinguish peaks with different amplitudes ${ }^{31}$. In Supplementary 262 Fig. 1, we show the radial Fourier profiles for various $m$ for a typical experiment (left column), 263 
and the raw radial profiles and how they are approximated by Eq. (2) (right column).

We also extracted the signal-to-noise ratio by comparing the standard deviation of the noise ${ }_{265}$ to the value of our signal. It is sufficiently high to exclude the possibility that the amplification we 266 observed is due to a noise fluctuation, and its contribution is negligible compared to other sources 267 of error.

PIV measurements. Close to the vortex core, the draining bathtub vortex is cylindrically sym- 269 metric to a good approximation. An appropriate choice of coordinates is, therefore, cylindrical 270 coordinates $(r, \theta, z)$. The velocity field will be independent of the angle $\theta$ and can be expressed as 271

$$
\mathbf{v}(r, z)=v_{r}(r, z) \mathbf{e}_{r}+v_{\theta}(r, z) \mathbf{e}_{\theta}+v_{z}(r, z) \mathbf{e}_{z}
$$

We are specifically interested in the velocity field at the free surface $z=h(r)$. When the free ${ }_{272}$ surface is flat, $h$ is constant and the vertical velocity $v_{z}$ vanishes. When the surface is not flat, 273 the $v_{z}$ component can be deduced from $v_{r}$ using the free surface profile $h(r)$ and the equation 274 $v_{z}(r, h(r))=\left.\left(\partial_{r} h\right) v_{r}\right|_{z=h}$. To obtain an estimate of $v_{z}$, we use a simple model for the free surface 275 shape $^{32}$,

$$
h(r)=h_{0}\left(1-\frac{r_{a}^{2}}{r^{2}}\right)
$$

where $h_{0}$ is the water height far from the vortex and $r_{a}$ is the radial position at which the free 277 surface passes through the sink hole. This approximation captures the essential features of our 278 experimental data. The components $v_{r}$ and $v_{\theta}$ are determined through the technique of Particle 279 Imaging Velocimetry (PIV), implemented through the Matlab extension PIVlab ${ }^{33,34}$. The tech- ${ }_{280}$ nique can be summarised as follows. 
The flow is seeded with flat paper particles of mean diameter $d=2 \mathrm{~mm}$. The particles ${ }^{282}$ are buoyant which allows us to evaluate the velocity field exclusively at the free surface. The 283 amount by which a particle deviates from the streamlines of the flow is given by the velocity lag 284 $U_{s}=d^{2}\left(\rho-\rho_{0}\right) a / 18 \mu$ (ref. $\left.{ }^{33}\right)$, where $\rho$ is the density of a particle, $\rho_{0}$ is the density of water, $\mu$ is ${ }_{285}$ the dynamic viscosity of water and $a$ is the acceleration of a particle. For fluid accelerations in our 286 system this is at most of the order $10^{-4} \mathrm{~m} / \mathrm{s}$, an order of magnitude below the smallest velocity in ${ }^{287}$ the flow. Thus we can safely neglect the effects of the velocity lag when considering the motions 288 of the particles in the flow.

The surface is illuminated using two light panels positioned at opposite sides of the tank. 290 The flow is imaged from above using a Phantom Miro Lab 340 high speed camera at a frame rate 291 of $800 \mathrm{fps}$ for an exposure time of $1200 \mu \mathrm{s}$. The raw images are analysed using PIVlab by taking a 292 small window in one image and looking for a window within the next image which maximizes the 293 correlation between the two. By knowing the distance between these two windows and the time 294 step between two images, it is possible to give each point on the image a velocity vector. This 295 process is repeated for all subsequent images and the results are then averaged in time to give a 296 mean velocity field.

The resulting velocity field is decomposed onto an $(r, \theta)$-basis centred about the vortex origin 298 to give the components $v_{r}$ and $v_{\theta}$. The centre is chosen so as to maximize the symmetry. In Fig. 4B 299 we show the norm of the velocity field on the free surface. We see that our vortex flow is symmetric 300 to a good approximation. To quantify the asymmetry of the flow, we estimate the coupling of waves 301 with $m \neq m^{\prime}$ through asymmetry. The change of the reflection coefficient due to this coupling is of 302 
the order of $\left|\tilde{v}^{l} / v_{g}\right|$, where $\tilde{v}^{l}$ is the angular Fourier component of azimuthal number $l=m-m^{\prime} . \quad{ }^{303}$ This ratio is smaller than $3 \%$ in all experiments. To obtain the radial profiles of $v_{r}$ and $v_{\theta}$, we 304 integrate them over the angle $\theta$. In Figs. 4C and 4D we show $v_{\theta}$ and the inward velocity tangent to 305 the free surface, $\tilde{v}_{r}=-\sqrt{v_{r}^{2}+v_{z}^{2}}$, as functions of $r$.

We compare the data for $v_{\theta}$ with the Lamb vortex ${ }^{32}$,

$$
v_{\theta}(r, h)=\frac{\Omega_{0} r_{0}^{2}}{r}\left[1-\exp \left(-\frac{r^{2}}{r_{0}^{2}}\right)\right],
$$

where $\Omega_{0}$ is the maximum angular velocity in the rotational core of characteristic radius $r_{0}$. (For ${ }_{308}$ $v_{\theta}$ we have $\Omega_{0}=69.4 \mathrm{rad} / \mathrm{s}$ and $r_{0}=1.34 \mathrm{~cm}$, and for $v_{r}$ we have $\Omega_{0}=-4.52 \mathrm{rad} / \mathrm{s}$ and 309 $r_{0}=1.39 \mathrm{~cm}$.) Outside the vortex core, this model reduces to the characteristic $1 / r$ dependence 310 of an incompressible, irrotational flow depending only on $r$. By observing that $v_{\theta}$ and $v_{r}$ exhibit 311 similar qualitative behaviour, $v_{r}$ is also fitted with a model of the form of equation (9). Figs. 4C 312 and 4D show that equation (9) captures the essential features of the measured velocity profiles. The ${ }_{313}$ angular velocity of the flow is given by $\Omega(r)=v_{\theta} / r$ which is shown in Fig. 4A. From this plot it 314 is clear that $\Omega$ reaches large enough values to be consistent with the detection of superradiance. 315

The data that support the plots within this paper and other findings of this study are available 316 from the corresponding author upon reasonable request.

31. Prabhu, K. Window functions and their applications in signal processing (CRC Press, 2013).

32. Lautrup, B. Physics of continuous matter: exotic and everyday phenomena in the macroscopic world (CRC press, 2011). 
33. Thielicke, W. The flapping flight of birds. Ph.D. thesis, University of Groningen (2014).

34. Thielicke, W. \& Stamhuis, E. PIVlab-towards user-friendly, affordable and accurate digital particle image velocimetry in MATLAB. Journal of Open Research Software 2, e30 (2014).

Acknowledgements We are indebted to the technical and administrative staff in the School of Physics \& Astronomy where our experimental setup is hosted. In particular, we want to thank Terry Wright and Tommy Napier for their support, hard work and sharing their technical knowledge and expertise with us to set up the experiment in Nottingham. Furthermore we would like to thank Bill Unruh, Stefano Liberati, Joseph Niemela, Luis Lehner, Vitor Cardoso, Michael Berry, Vincent Pagneux, Daniele Faccio, Fedja Orucevic, Jörg Schmiedmayer and Thomas Fernholz for discussions regarding the experiment, and we wish to thank Michael Berry, Vitor Cardoso, Daniele Faccio, Luis Lehner, Stefano Liberati, and Bill Unruh for comments on the paper. Although all experiments have been conducted at The University of Nottingham, the initial stages of the experiment took place at ICTP/SISSA in Trieste (Italy) and would not have been possible without the support by Joseph Niemela, Stefano Liberati and Guido Martinelli. S. W. would like to thank Matt Penrice, Angus Prain, Miltcho Danailov, Ivan Cudin, Henry Tanner, Zack Fifer, Andreas Finke, and Dylan Russon for their contributions at different stages of the experiment. S. W. would also like to thank Thomas Sotiriou for the many discussions on all aspects of the project.

A. C. acknowledges funding received from the European Union's Horizon 2020 research and innovation programme under the Marie Sklodowska Curie grant agreement No 655524. M. R. acknowledges financial support from the São Paulo Research Foundation (FAPESP), Grants No. 2005/04219-0, No. 2010/201231, No. 2013/09357-9, No. 2013/15748-0 and No. 2015/14077-0. M. R. and Ted T. are also grateful to S. W. and the University of Nottingham for hospitality while this work was being completed. S. W. ac- 
knowledges financial support provided under the Royal Society University Research Fellow (UF120112), the Nottingham Advanced Research Fellow (A2RHS2), the Royal Society Project (RG130377) grants and the EPSRC Project Grant (EP/P00637X/1). The initial stages of the experiment were funded by S. W. 's Research Awards for Young Scientists (in 2011 and 2012) and by the Marie Curie Career Integration Grant (MULTI-QG-2011).

Author Contributions All authors contributed substantially to the work.

Author Information The authors declare that they have no competing financial interests. Correspondence and requests for materials should be addressed to S.W. (email: silke.weinfurtner@ nottingham.ac.uk). 

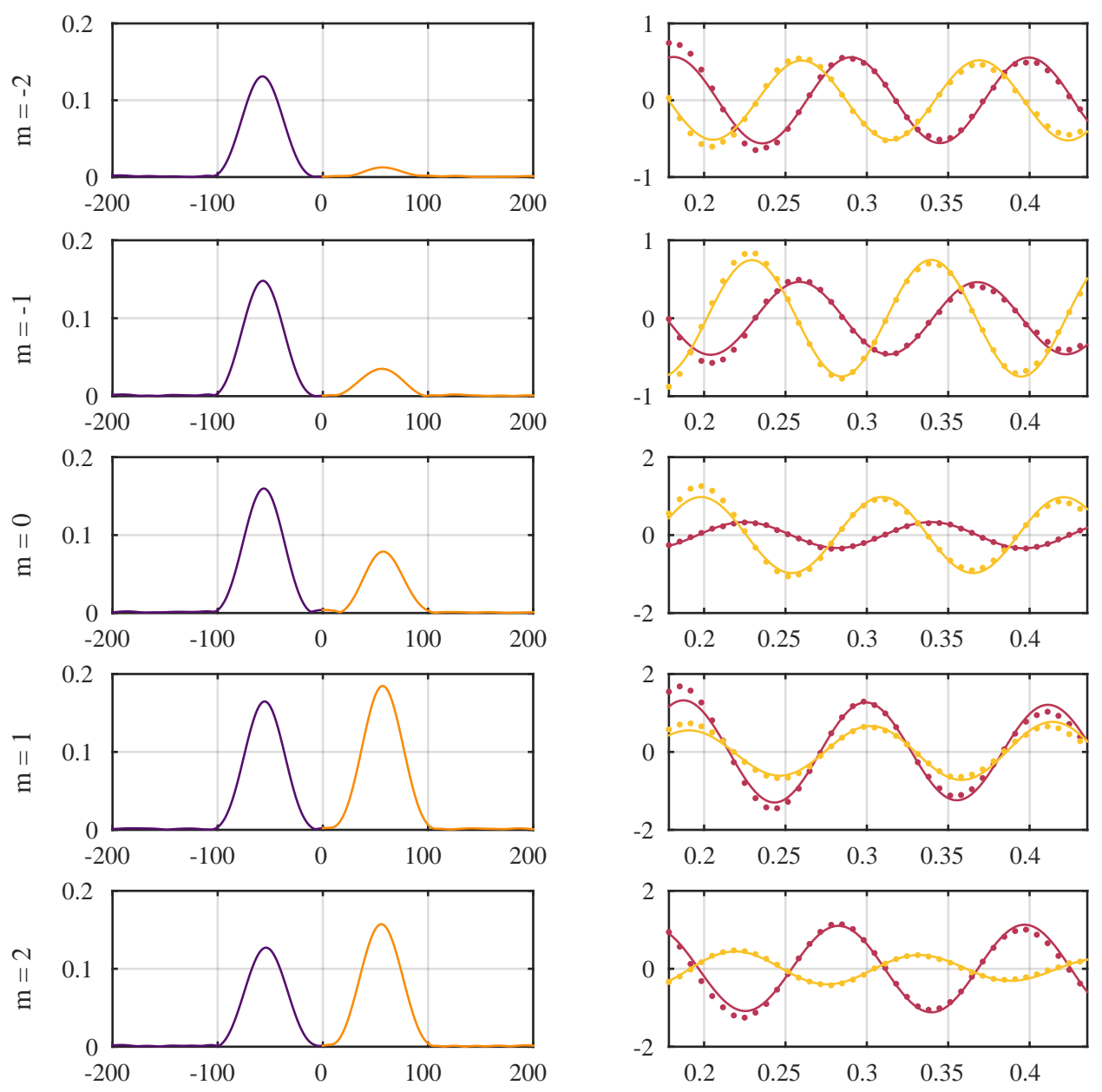

Supplementary Figure $1 \mid$ Left side: Modulus of the Fourier profiles $\left|\tilde{\varphi}_{m}(k)\right|^{2}$ for various $m$. Right side: Radial profiles $\varphi_{m}(r)$ for various $m$ (maroon: real part, yellow: imaginary part). The vertical axis is in arbitrary units. The horizontal axes in inverse metres $\left(\mathrm{m}^{-1}\right)$ on the left side, and metres $(\mathrm{m})$ on the right side. The dots are the experimental data (for clarity, only 1 out of 3 is represented), and the solid lines show the approximation of Eq. (2) for the extracted values of $A_{\text {in }}$ and $A_{\text {out }}$. 